
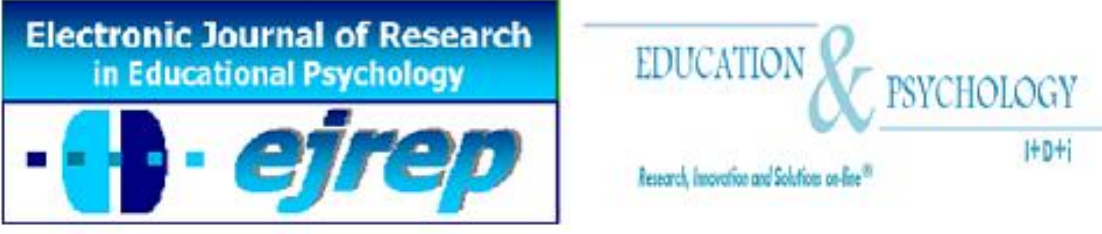

\title{
Validez Factorial de un Cuestionario para Evaluar la Percepción Inicial de los Estudiantes Universitarios sobre la Evaluación de los Aprendizajes
}

\author{
Fernando Doménech Betoret ${ }^{1}$ \\ $\mathbf{y}$ \\ Miguel Angel Fortea Bagán ${ }^{2}$
}

${ }^{1}$ Psicología Evolutiva y de la Educación, Universitat Jaume I, Castellón ${ }^{2}$ Unitat de Suport Educatiu (USE), Universitat Jaume I, Castellón

\section{España}

Correspondencia: Dr. Fernando Doménech Betoret, Dept. de Psicología Evolutiva y de la Educación, Universitat Jaume I, Campus Riu Sec, 12071 Castellón (Spain), E-mail: betoret@psi.uji.es, Tel.: +34964 7295 50, Fax: +34964729262

(C) Education \& Psychology I+D+i and Ilustre Colegio Oficial de Psicología de Andalucía Oriental (Spain) 


\section{Resumen}

Introducción. La investigación educativa ha comprobado ampliamente que la percepción del estudiante sobre el sistema de evaluación de la asignatura va a jugar un papel fundamental en su implicación (enfoque profundo vs. enfoque superficial) en el proceso de enseñanza/aprendizaje de la asignatura. El objetivo del presente trabajo consistió en examinar la validez factorial y fiabilidad de una escala para evaluar la percepción de los estudiantes universitarios, al inicio de curso, sobre el sistema de evaluación de la asignatura que van a cursar.

Método. La escala fue creada a partir de los debates y reflexiones llevados a cabo en grupos de discusión de un seminario sobre evaluación educativa desarrollado en la Universitat Jaume I, organizado por la Unitat de Suport Educatiu (USE) durante los cursos 2012/13 y 2013/14, y en el que participaron un total de 18 profesores/as que impartían docencia en diferentes asignaturas y titulaciones. El cuestionario se aplicó a una amplia muestra de 435 estudiantes universitarios de diferentes asignaturas y titulaciones.

Resultados. Los resultados obtenidos a través de un análisis factorial confirmatorio y del test alpha de Cronbach revelaron: a) que el cuestionario posee una estructura tridimensional, es decir, está formado por tres factores independientes que se refieren a tres constructos importantes relacionados con la calidad evaluativa de los aprendizajes y b) que posee buenas propiedades psicométricas de validez y fiabilidad.

Conclusión. Las implicaciones prácticas que se pueden derivar de la utilización de este instrumento en el contexto universitario se discuten en el artículo.

Palabras clave: Evaluación universitaria, Evaluación de los aprendizajes universitarios, Calidad evaluativa, Reflexión docente, Escala de evaluación.

Recibido: 16.04.15 Aceptación Inicial: 21.05.15 Aceptación final: 06.11.15 


\title{
Factorial Validity of a Questionnaire to Evaluate University Students'́ Initial Perception of Learning Evaluation.
}

\begin{abstract}
Introduction. Education research has clearly verified that a student's perception of the system evaluating the subject matter will play a fundamental role in its implication (deep approach vs. surface approach) in the teaching/learning process of the subject matter. The present work aims to examine the factorial validity and reliability of a scale to evaluate university students' perceptions when the academic year starts of the evaluation system of the subject matter to be taught.

Method. A scale was created from debates organised with and reflections made in discussion groups during a seminar on education evaluation at the Universitat Jaume I. It was run by the Unitat de Suport Educatiu (Education Support Unit) in academic years 2012/13 and 2013/14, and 18 teachers participated who taught the various subject matters and degrees. A questionnaire was administered to a study sample of 435 university students studying different matters and degrees.

Results. The results obtained from a confirmatory factorial analysis and Cronbach's alpha revealed that: a) the questionnaire had a three-dimensional structure; that is, it consisted in three independent factors referring to three important constructs relating to the evaluative quality of learning; b) it had good psychometric properties for validity and reliability.

Conclusion. The practical implications that can derive from using this instrument in the university context are discussed in this article.

Keywords: University evaluation, University learning evaluation, Evaluative quality, Teaching reflection, Evaluation scale.
\end{abstract}




\section{Introducción}

Con la implantación de los nuevos planes de estudio en la universidad española propiciados por el Espacio Europeo de Educación Superior (EEES), se ha producido a la vez un cambio de paradigma educativo que ha implicado una nueva forma de enseñar y de evaluar en la educación superior. Los tipos de evaluación pueden ser muy variados y diferentes (evaluación formativa-continua, sumativa-final, diagnóstica, etc.) y, según SanMartí (2007), los mejores resultados se obtienen cuando se usan de forma combinada y estratégica, y ayudan al estudiante a regular su propia actividad de aprendizaje. Tradicionalmente la evaluación en la universidad ha estado centrada en los resultados, sin embargo, desde el EEES se propugna una evaluación más centrada en el proceso que en los resultados, subrayando la importancia de llevar a cabo una evaluación formativa-continua integrada en el proceso de enseñanza/aprendizaje.

La evaluación constituye el elemento de la actividad docente que más repercusiones tiene sobre los estudiantes (Zabalza, 2003). La percepción que los estudiantes tengan de la evaluación les puede afectar de muchas formas (Álvarez, 2008; Doménech, 2011; Struyven, Dochy, \& Janssens, 2005) como por ejemplo, en su forma de estudiar (Balch, 2007; Scouller, 1998; Tian, 2007), en su motivación o intención de aprender (Alonso, 2005), en sus expectativas (Doménech, 2011), y en definitiva en su éxito académico. Además, toda la estructura curricular (objetivos, contenidos, metodología, actividades, etc.) pivota sobre el eje de la evaluación (Zabalza, 2003).

Existen también numerosos estudios empíricos que han puesto de manifiesto la relación entre evaluación esperada y en su dedicación e implicación, adoptando un enfoque de aprendizaje profundo vs. superficial (tipología de Marton y Saljo, 1976). La investigación educativa ha comprobado ampliamente que en función de cómo el estudiante perciba que va a ser evaluado adoptará un enfoque de aprendizaje u otro. Los estudiantes adoptarán aquel enfoque que mejor responda y las demandas de la asignatura y de la evaluación (Kember, 2000). El enfoque adoptado por el estudiante influirá en la calidad del aprendizaje alcanzado, ya que desarrollará un tipo de capacidades/habilidades u otras. El enfoque profundo desarrollará capacidades de alto nivel (como por ejemplo: análisis, resolución de problemas y pensamiento crítico), mientras que el enfoque superficial desarrollará capacidades de bajo nivel (como por ejemplo: memorización y comprensión deficiente). 
Los estudios anteriores constatan la importancia que tiene en el aprendizaje la percepción de los estudiantes se forman sobre cómo van a ser evaluados, pero dicha percepción no sólo va a depender de la evaluación planteada por el profesor en el programa de la asignatura, sino también de su forma de transmitirla para que no existan dudas o malentendidos. Además, los estudiantes tienen derecho a saber cómo van a ser evaluados desde el inicio de curso. Según Morales (2009), los profesores antes de comenzar el curso suelen pensar en el programa, en sus temas, en cómo van a organizar su docencia, mientras que en la evaluación suelen pensar al final de curso. En cambio, los estudiantes suelen seguir un proceso más o menos inverso, a ellos lo primero que les interesa conocer es la evaluación de la asignatura y a partir de ahí organizan su estudio. Por tanto, lo correcto por parte del profesorado sería reflexionar, al inicio de curso, sobre cómo van a evaluar, introducir su propuesta de evaluación en el programa de la asignatura e informar a las estudiantes, y a continuación comprobar la percepción que se han formado esos estudiantes, ya que dicha percepción va a condicionar desde el inicio su aprendizaje.

Organismos y autores que citamos a continuación justifican la necesidad de dar a conocer a los estudiantes cómo va a ser el proceso evaluativo del profesor, así como sobre los derechos y deberes derivados de la normativa vigente universitaria sobre evaluación, que les pueda interesar o afectar. Así, en el contexto del Espacio Europeo de Educación Superior (EEES), la European Association for Quality Assurance in Higher Education (ENQA) (2005) respecto a la calidad de la "evaluación de los estudiantes" establece que éstos deben ser evaluados utilizando criterios, normativas y procedimientos de evaluación que se hayan publicado (y que además se apliquen de manera coherente):

Se debería proporcionar a los estudiantes información clara sobre la estrategia de evaluación que está siendo utilizado en su programa, sobre los métodos de examen y evaluación a los que serán sometidos, sobre lo que se espera de ellos y sobre los criterios que se aplicarán para la evaluación de su actuación (ENQA, 2005, p. 18).

Por otra parte, autores como Padilla y Gil (2008) resaltan que es necesario que los estudiantes conozcan los criterios e indicadores que se van a utilizar para valorar su aprendizaje, ya que suele ser habitual que estos criterios sean implícitos y que el docente los utilice sin informar de ellos. No son pocas las situaciones en que los estudiantes denuncian que sistemas de evaluación en la universidad son subjetivos, aplicados de forma arbitraria e incluso con criterios de superación de la asignatura confusos y vagos que los dejan indefensos ante las posibles interpretaciones que haga el profesorado. De hecho, el primer Estatuto del 
Estudiante Universitario aprobado en la legislación española (BOE RD1791/2010) ha establecido el derecho del estudiante a una evaluación continua y objetiva y más concretamente en su artículo 25.1:

La evaluación del rendimiento académico de los estudiantes responderá a criterios públicos y objetivos y tenderá hacia la evaluación continua, entendida como herramienta de corresponsabilidad educativa y como un elemento del proceso de enseñanza-aprendizaje que informa al estudiante sobre su proceso de aprendizaje (BOE. RD 1791/2010, p. 109365).

De los argumentos presentados se derivan las siguientes conclusiones: a) Evaluación y calidad van muy unidas, b) La manera más eficaz de cambiar la forma de estudiar de los alumnos y mejorar la calidad del aprendizaje es cambiando el sistema de evaluación de la asignatura y c) La información y explicación que proporcione el profesor, al inicio de curso, a los estudiantes sobre el sistema de evaluación de su asignatura, así como de determinadas cuestiones derivadas de la normativa universitaria vigente que les puede afectar en el tema de la evaluación, van a jugar un papel fundamental.

Basándonos en las consideraciones anteriores, el objetivo de la presente investigación consiste en poner al servicio del profesorado universitario una herramienta capaz de diagnosticar, al inicio de curso, la percepción que se han formado sus estudiantes sobre la evaluación de la asignatura. Específicamente, se pretende validar un instrumento de medida que hemos denominado "cuestionario para la evaluación de la percepción inicial de los estudiantes universitarios sobre el sistema de evaluación de la asignatura" (CEPISEA) formado por tres componentes: a) Cómo los estudiantes perciben al inicio del curso la propuesta de evaluación formulada por el profesor, b) Explicación de la evaluación por el profesor, y c) Información sobre la evaluación de la asignatura a través de los procedimientos establecidos (clase presencial, aula virtual y libro electrónico de la universidad). La aplicación de dicho cuestionario al principio de curso no sólo fomentará la reflexión y el debate entre profesor y estudiantes sobre la evaluación de la asignatura sino que además proporcionará al profesor información valiosa sobre la percepción de los estudiantes acerca del sistema de evaluación de la asignatura que se va a impartir, lo que permitirá identificar deficiencias e introducir mejoras desde principio de curso. 


\section{Método}

\section{Participantes}

Una amplia muestra de 435 estudiantes universitarios participaron en este estudio. La muestra estaba formada por $138(31,7 \%)$ hombres y $297(68,3 \%)$ mujeres, que cursaban estudios en diferentes asignaturas y titulaciones en la Universitat Jaume I de Castellón, situada en el este de España. La edad de los estudiantes oscilaba entre 17 y 65 años de edad $($ media $=21,28$, D.T. $=4,83)$.

\section{Instrumentos: elaboración}

El cuestionario para la evaluación de la percepción inicial de los estudiantes universitarios sobre el sistema de evaluación de la asignatura (CEPISCEA) utilizado se elaboró en un seminario de evaluación educativa universitaria, desarrollado durante los cursos 2012/13 y 2013/14, por la Unitat de Suport Educatiu (USE) de la Universitat Jaume I. El cuestionario se aplicó en las asignaturas impartidas por los propios docentes asistentes al seminario. Sin suponer un muestreo estratificado de la docencia de la universidad, sí que garantiza cierta variabilidad de los datos: 5 asignaturas de la Escuela superior de tecnología $(\mathrm{N}=105), 3$ de la facultad de humanas y sociales $(\mathrm{N}=152), 1$ de ciencias jurídicas $(\mathrm{N}=42)$ y 2 de ciencias de la salud $(\mathrm{N}=136)$. De esta forma, se han incluido asignaturas de 8 grados diferentes y de 1 master. Queremos señalar que, en cumplimiento con las directrices del seminario, cada profesor recibió un informe con los resultados obtenidos de la aplicación del cuestionario en sus respectivas asignaturas, con el objetivo de que los pudiese comentar libremente con sus estudiantes. Los resultados globales se discutieron en el seminario permanente.

Como ya se ha indicado en el párrafo anterior, el cuestionario (CEPISEA) original se diseñó durante el desarrollo de un seminario de evaluación universitaria, a partir de la revisión de la bibliografía sobre evaluación educativa, del análisis de los indicadores de la European Association for Quality Assurance in Higher Education (ENQA, 2005) y de las reflexiones y debates generados en los grupos de discusión que se crearon en el seminario. Las propiedades psicométricas de la escala original, resultante de los grupos de discusión del seminario, ya fueron examinadas en un estudio piloto previo (Doménech, Fortea, \& Benaloy, 2013).

En el presente estudio hemos aplicado el cuestionario depurado, obtenido a partir del estudio piloto anterior, a una muestra más extensa de estudiantes universitarios de diferentes 
asignaturas (pertenecientes a diferentes titulaciones) impartidas por los propios docentes asistentes al seminario. El cuestionario actual examinado (ver anexo) está formado por un total de 25 ítems $(\alpha=0.916)$. La respuesta de los estudiantes se puntuó utilizando una escala tipo likert formada por cinco opciones, ordenadas de 1 (totalmente en desacuerdo) a 5 (totalmente de acuerdo). Los 25 ítems que conforman el cuestionario se estructuran en 3 bloques. Bloque 1 ( 8 ítems, $\alpha=0.816$ ): Evaluación de la información pública del sistema de evaluación de la asignatura (guía docente oficial); Bloque 2 (7 ítems, $\alpha=0.855$ ): Evaluación sobre cómo se ha transmitido la información al estudiante por parte del profesorado; y Bloque 3 (10 ítems, $\alpha=0.871$ ) Percepción del estudiante del sistema de evaluación de la asignatura ante la información recibida.

Al final del cuestionario se incluyen dos ítems adicionales para explorar las expectativas de los estudiantes (de satisfacción con la evaluación de la asignatura y de resultado en función de la evaluación percibida), puntuados con una escala Likert de 1 a 10, si bien la información aportada por estos dos ítems no será considerada en el presente estudio. El cuestionario se completaba solicitando al estudiante que nos ayudase a mejorar el sistema de evaluación de la asignatura, contestando por escrito, aquellas cuestiones, que en su opinión, podían ser susceptibles de mejora. El cuestionario completo y detallado se puede ver en el anexo.

Este instrumento se ha diseñado tratando de cumplir una serie de requisitos que faciliten su aplicación en el ámbito universitario y con la finalidad de contribuir a la mejora de la práctica evaluativa de los docentes:

1) Útil para cualquier tipo de docencia universitaria

2) Rápido de suministrar (con solo 25 ítems se contesta en menos de 10 minutos)

3) Fácil de corregir y explotar por los propios docentes de la asignatura.

4) Se puede suministrar/usar desde los primeros días de clase.

5) Se recoge información poco "sensible" o "comprometedora" (es anónimo y la información está centrada más en "procesos” que en el propio profesor).

6) Se facilita la reflexión con los estudiantes desde el inicio del curso.

\section{Procedimiento}

El cuestionario CEPISEA fue aplicado, en formato de papel y lápiz, durante el curso 2013/14 en las aulas universitarias de 12 asignaturas diferentes, a cargo de un investigador que leyó las instrucciones y recogió los cuestionarios cumplimentados, mientras el profesor o 
profesora de la asignatura permanecía fuera del aula (para no influir en las evaluaciones). El investigador tenía la consigna de no hacer aclaraciones sobre la formulación de los ítems del cuestionario. Ante cualquier problema de comprensión, el ítem debía dejarse sin responder y hacerse constar en un apartado final del cuestionario, reservado para introducir los comentarios al respecto (nos interesaba saber si existían dificultades de comprensión en alguno de los ítems). Finalmente respondieron el 100\% de los estudiantes presentes en el aula en el momento del pase del cuestionario. En total participaron 435 estudiantes de forma anónima, cuyas respuestas fueron válidas en todos los casos. Cada profesor y profesora recibió un informe individual con los resultados obtenidos en su asignatura.

\section{Análisis de datos}

Se llevó a cabo un análisis factorial confirmatorio (AFC) a través del programa EQS (Bentler, 1995, 2006) para comprobar la bondad de ajuste del modelo de medida hipotetizado. Se utilizó es método de estimación de Máxima Verosimilitud (ML), y ML, Robusto (por si no existiese normalidad multivariante). Ya que el valor de chi-cuadrado es sensible al tamaño de la muestra, los expertos recomiendan utilizar otros índices de ajuste tales como CFI, NNFI y RMSA (Bentler, 1990). Valores de RMSEA por debajo de .05 indican un ajuste óptimo, mientras que valores por encima de .08 indican un mal ajuste (Browne \& Cudeck, 1993). Valores por encima de .90 indican un buen ajuste para NNFI y CFI (Hoyle, 1995). También se suele utilizar el valor del cociente resultante de dividir chi-cuadrado entre los grados de libertad ( $\chi 2 /$ d.f.), indicando un buen ajuste cuando dicho índice es igual a 3 o inferior (Hoe, 2008).

\section{Resultados}

\section{Descriptivos de las tres dimensiones del cuestionario por sexo}

En primer lugar se calcularon las medias y desviaciones típicas de los tres factores que componen el cuestionario, para toda la muestra de sujetos y también agrupados por sexo. Los resultados indican que las mujeres otorgaron puntuaciones más altas en las tres dimensiones del cuestionario. La prueba $\mathrm{t}$ de Student realizada constató la existencia de diferencias significativas por sexo en las tres dimensiones. Los resultados detallados se presentan en la Tabla 1. 
Tabla 1. Descriptivos de los factores y prueba t de Student de contraste de medias por sexo.

\begin{tabular}{lcccc}
\hline Factores & $\begin{array}{c}\text { M(D.T) } \\
\text { Global }\end{array}$ & $\begin{array}{c}\text { M(D.T) } \\
\text { Hombres }\end{array}$ & $\begin{array}{c}\text { M (D.T) } \\
\text { Mujeres }\end{array}$ & $t$ \\
\hline $\begin{array}{l}\text { F1: Información programa (de item 1 a item 8; } \alpha=0.81) \\
\text { Se han asumido varianzas iguales }\end{array}$ & $3,57(0,57)$ & $3,41(0,70)$ & $3,64(0,57)$ & \\
No se han asumido varianzas iguales & & & & $-3,628^{* *}$ \\
F2: Explicación evaluación (de item 9 a item 15; $\alpha=0.85)$ & $4,10(0,65)$ & $3,94(0,71)$ & $4,17(0,61)$ & $-3,374^{* *}$ \\
Se han asumido varianzas iguales & & & & $-3,428^{* *}$ \\
No se han asumido varianzas iguales & & & & $-3,256^{* *}$ \\
F3: Percepción de propuesta evaluadora (de item 16 a item & $3,76(0,62)$ & $3,51(0,62)$ & $3,88(0,59)$ & \\
25; $\alpha=0.87)$ & & & & $-5,923^{* *}$ \\
Se han asumido varianzas iguales & & & & $-5,813^{* *}$ \\
No se han asumido varianzas iguales & & & & \\
\hline
\end{tabular}

$* p<.05 \quad * * p<.01$

\section{Análisis correlacional de los ítems del cuestionario}

A continuación se realizó un análisis correlacional bivariado de Pearson para explorar las relaciones entre los ítems que conforman el cuestionario. Los resultados se muestran en la Tabla 2. Como se puede observar aparecen correlaciones importantes entre los ítems del mismo bloque, lo cual parece indicar que, en general, los ítems están bien encuadrados en la configuración pronosticada. También queremos señalar que la mayoría de las correlaciones entre la variable sexo y los ítems del cuestionario han resultado ser significativas.

\section{Análisis factorial confirmatorio}

Se llevó a cabo un análisis factorial confirmatorio (AFC) para verificar la estructura factorial del modelo de medida hipotetizado. Los índices obtenidos tras la eliminación de ítem 7 del bloque 1 (siguiendo las recomendaciones de los test de Wald y Lagrange del programa EQS para el ajuste de parámetros) fueron los siguientes, utilizando el método de ML para la estimación de parámetros: $\chi^{2}=626.316$ basado en 249 d.f., $\mathrm{p}=.000$; Bentler-Bonett Non Normed Fit Index $(\mathrm{BNNFI})=.892$; Comparative Fit Index $(\mathrm{CFI})=.901$; Root Mean-Square Error of Approximation $(\mathrm{RMSEA})=.062 ; \mathrm{y} \chi^{2} /$ d.f. $=2.51$. Con el método ML, Robusto se obtuvieron los siguientes índices: $\chi^{2}$ (Satorra-Bentler scaled chi-square) $=502.327$ basado en 249 d.f., p=.000; Bentler-Bonett Non Normed Fit Index $(B N N F I)=.904$; Comparative Fit Index $(\mathrm{CFI})=.913$; Root Mean-Square Error of Approximation $(\mathrm{RMSEA})=.050 ; \mathrm{y} \chi^{2} / \mathrm{d} . \mathrm{f} .=$ 2.01. Los índices mostraron un buen ajuste del modelo a los datos empíricos apoyando la estructura tridimensional del cuestionario. La Figura 1 muestra la configuración estructural y los coeficientes estandarizados del modelo de medida obtenido. 


\section{Tabla 2. Correlaciones bivariadas de Pearson entre los ítems que conforman el cuestionario.}

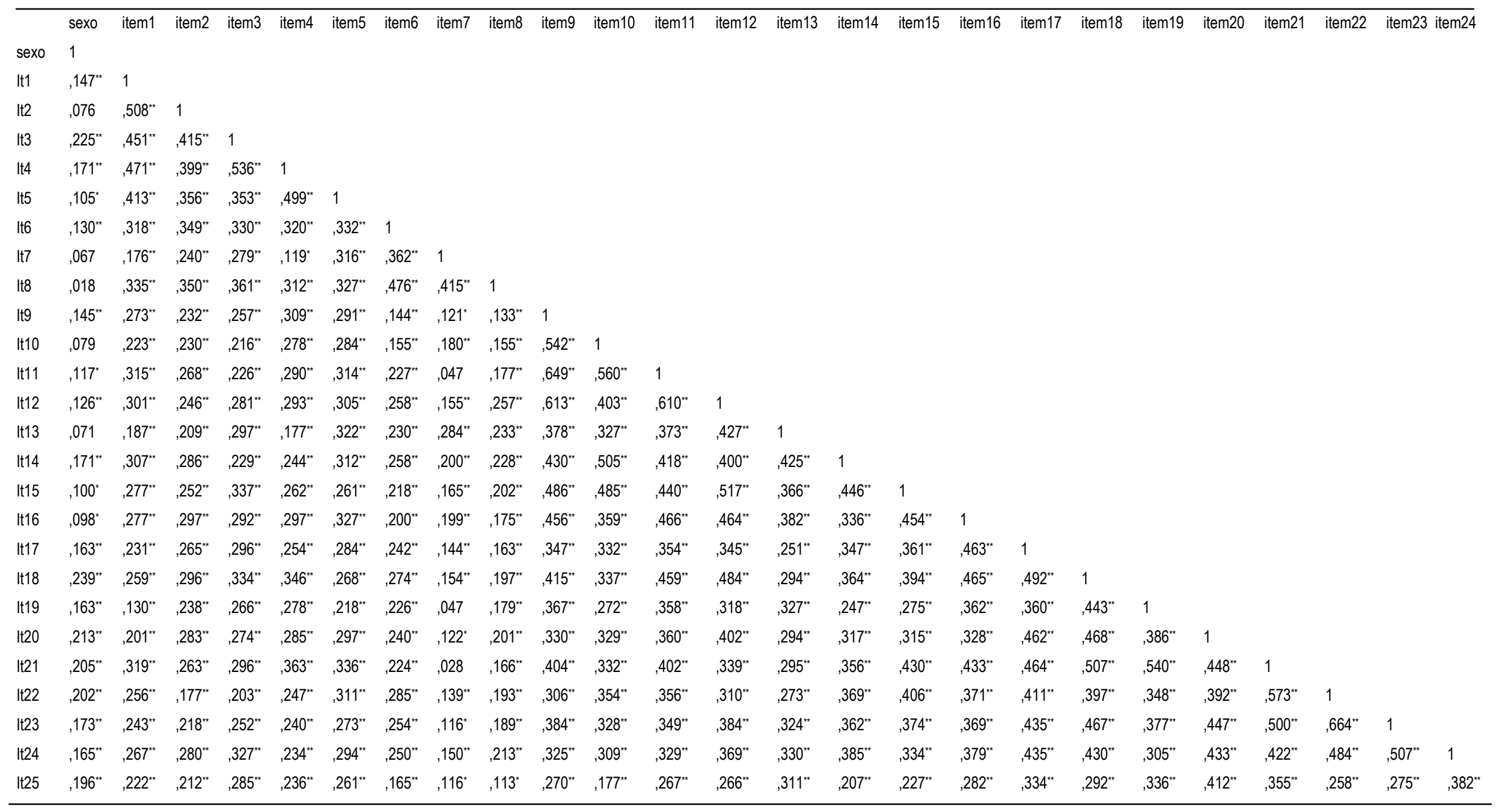

$* p<.05 \quad * * p<.01 ; \quad$ Sexo: 1 (hombre), 2 (mujer) 


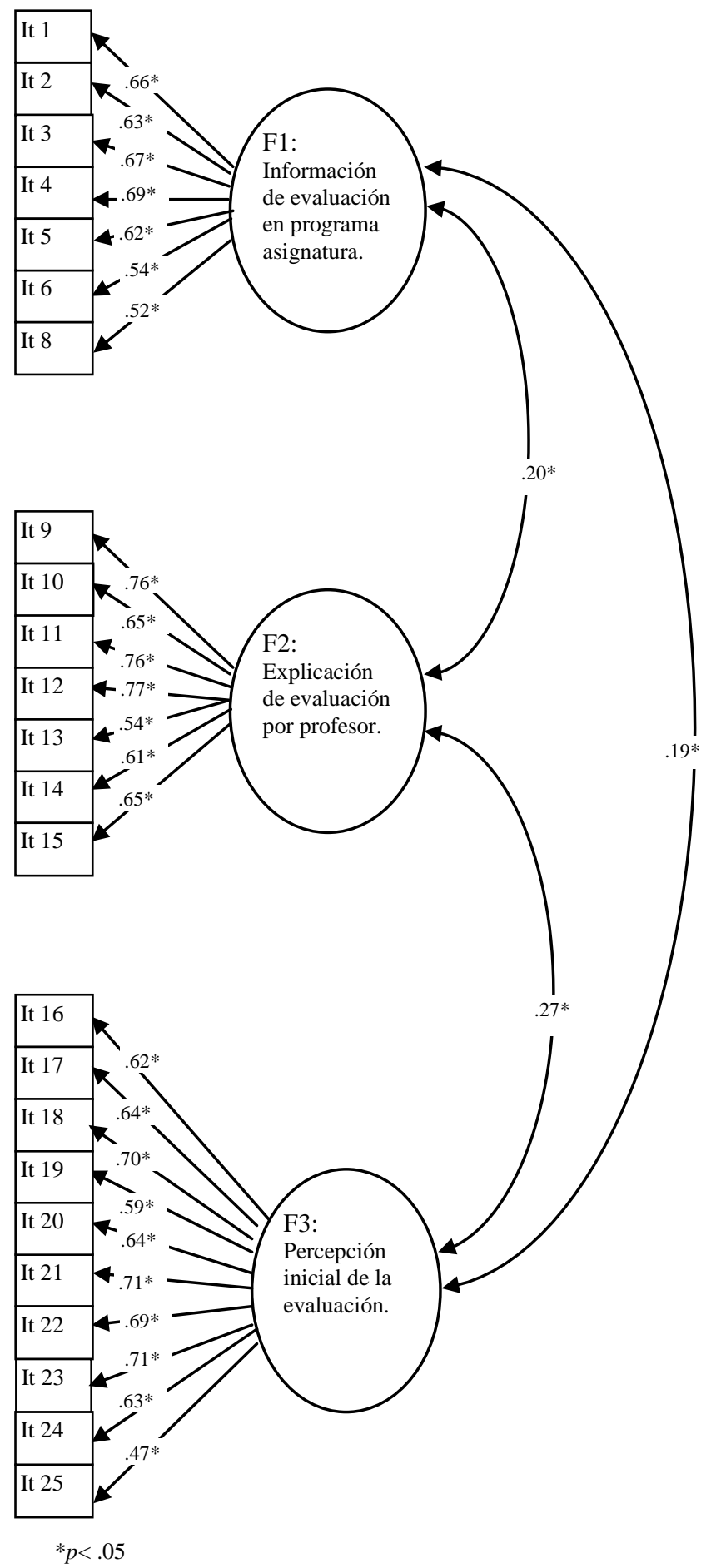

Figura 1. Configuración estructural y coeficientes estandarizados del modelo de medida optimizado obtenido tras la eliminación del ítem 7 (F1), siguiendo las recomendaciones de los test de Wald y Lagrange para quitar y añadir parámetros del programa EQS.

Nota. Las variables latentes representan:

F1 (7 ítems, $\alpha=0.816$ ): El programa oficial o guía docente ECTS de la asignatura (publicado en el LLeu o libro electrónico de la universidad) proporciona suficiente información sobre...

F2 (7 ítems, $\alpha=0.855$ ): El profesorado se ha esforzado por transmitir y explicar al estudiante el sistema de evaluación de la asignatura...

F3 (10 ítems, $\alpha=0.871)$ : Cómo se percibe al inicio del curso el sistema de evaluación. La evaluación que se propone para esta asignatura me parece... 


\section{Discusión y conclusiones}

El principal objetivo del presente estudio consistía en probar la validez factorial y fiabilidad del "cuestionario para la evaluación de la percepción inicial de los estudiantes universitarios sobre el sistema de evaluación de la asignatura" (CEPISEA). La prueba $t$ de Student inicial realizada para contrastar la percepción formada por estudiantes masculinos y femeninos constató la existencia de diferencias significativas por sexo en las tres dimensiones del cuestionario. Los resultados indicaron que la percepción inicial que se formaron las mujeres del sistema de evaluación de las asignaturas fue significativamente más positiva que la que se formaron los hombres. Aunque este resultado hay que tomarlo con cautela debido a la descompensación de la muestra para ambos grupos de sujetos (138 hombres y 297 mujeres), es un dato interesante a tener en cuenta, ya que en función de que en la clase predomine un género u otro podría influir en el resultado global de las evaluaciones.

Los resultados obtenidos a través del análisis factorial confirmatorio revelaron que el modelo de medida hipotetizado se ajustó bien a los datos empíricos, tras eliminar un ítem del cuestionario inicial (ítem 7, F1), lo cual indica que el planteamiento teórico de la escala era adecuado. Por otra parte, los datos confirmaron la estructura tridimensional del cuestionario, es decir, formado por tres factores independientes que se refieren a tres constructos importantes relacionados con la calidad evaluativa de los aprendizajes: A) El programa oficial o guía docente ECTS de la asignatura (publicado en el LLEU) proporciona suficiente información sobre ...... (el LLEU, o libro electrónico de la universidad, es como se denomina en nuestra universidad al espacio web donde se publica la información oficial de todas las titulaciones y las respectivas Guías ECTS de las asignaturas); B) El profesorado se ha esforzado por transmitir y explicar al estudiante el sistema de evaluación de la asignatura ......; y C) Cómo se percibe al inicio del curso el sistema de evaluación. La evaluación que se propone para esta asignatura me parece...... Además, los valores obtenidos a través de los análisis de fiabilidad, a través del test alpha de Cronbach, indicaron que tanto la escala global como las tres subescalas que la componen presentan buenos índices de consistencia interna (según el criterio establecido por Nunnaly \& Berstein, 1994). En resumen, teniendo en cuenta los resultados obtenidos podemos afirmar que el instrumento analizado posee buenas propiedades psicométricas de validez y fiabilidad para poder ser aplicado con garantías en el contexto educativo universitario. La escala final definitiva se presente en el anexo. 
La escala propuesta puede resultar una herramienta útil para evaluar la percepción que se forman los estudiantes al principio de curso sobre la propuesta evaluativa planteada por el profesorado universitario en sus respectivas asignaturas. Esta información obtenida a principio de curso es muy valiosa para el profesorado ya que le incita a la reflexión de su propia propuesta evaluadora desde el inicio del proceso de enseñanza-aprendizaje (Doménech, 2013). Dicha reflexión facilitará la detección de posibles deficiencias y contribuirá a mejorar la calidad evaluativa de los aprendizajes, además de suponer un punto de arranque para implementar una evaluación compartida (López, 2009). También existe otro argumento poderoso, asumido por la mayoría de los autores, para utilizar este tipo de instrumento al principio de curso: "Los estudiantes aprenden en función de cómo perciben que van a ser evaluados". La “calidad del aprendizaje" está determinada por el enfoque adoptado por el estudiante para aprender, y éste a su vez dependerá de cómo los estudiantes perciban que van a ser evaluados (Entwistle, 1987; Marton, 1988; Snow \& Swanson, 1992). Por tanto, es importante conocer la percepción que tienen los estudiantes sobre cómo son evaluados (ver Maclellan, 2001; Struyven, Dochy, \& Janssens, 2005; Struyven, Trillo, \& Porto, 1999), lo que permitirá activar mecanismos para mejorar la percepción de los estudiantes desde el inicio, lo que, a su vez, influirá en la mejora de la satisfacción y del aprendizaje. También puede contribuir a mitigar la elevada ansiedad que sufren los estudiantes universitarios frente a los exámenes (Álvarez, Aguilar y Lorenzo, 2012).

En futuros estudios sería recomendable realizar un estudio comparativo de contraste de medias para muestras independientes, agrupando a los estudiantes por sexo y facultad (con muestras más extensas y equilibradas), para examinar si dichas variables pueden tener un efecto modulador en la percepción de los estudiantes sobre la evaluación de los aprendizajes. También son necesarios futuros estudios adaptar el instrumento a otros niveles educativos y a otros contextos culturales (estudiantes de otros países), así como examinar su estabilidad en contextos similares. 


\section{Agradecimientos*}

*El presente trabajo surge en el ámbito de un Seminario Permanente de Innovación Educativa de la Universitat Jaume I (UJI), conformado por un grupo de 16 docentes universitarios, una estudiante y un técnico experto en psicología educativa, que se reúnen periódicamente para debatir y reflexionar sobre la evaluación continua en el contexto del Espacio Europeo de Educación Superior. Desde el curso 2012/13, dicho seminario está implementando un proyecto financiado en la convocatoria de innovación educativa propia de la UJI con el título "Evaluación educativa y aprendizaje de calidad un binomio inseparable".

Miembros del Seminario: Andrio Balado, Andreu; Badenas Carpio, Jorge; Flores Buils, Raquel; García De Toro, Ana Cristina; Górriz Plumed, Ana Belén; Gozalbo Nebot, Ana; Huedo Dorda, Patricia Margarita; Molés Cases, Teresa; Peydró Pons, Laura; Ruiz Bernardo, Ma Paola; Sánchez Vilches, Enrique Javier; Sanz Solana, Vicente; Seguí Cosme, Salvador; Vellón Lahoz, Francisco Javier; Vila Pastor, Carlos; Benaloy Expósito, Ma Dolores; Fortea Bagán, Miguel Ángel y Doménech Betoret, Fernando

\section{Referencias}

Alonso, J. (2005). La motivación para el aprendizaje: La perspectiva de los alumnos. En Ministerio de Educación y Ciencia (ed.), La orientación escolar en centros educativos, 209-242. Madrid: MEC.

Álvarez, I. (2008). Evaluación del aprendizaje en la universidad: Una mirada retrospectiva y prospectiva desde la divulgación científica. Revista Electrónica de Investigación Educativa, 14, 6(1), 235-272.

Álvarez, J., Aguilar, J. M., Lorenzo, J. J. (2012). La Ansiedad ante los Exámenes en Estudiantes Universitarios: Relaciones con variables personales y académicas. Electronic Journal of Research in Educational Psychology, 10(1), 333-354

Balch, W. R. (2007). Effects of Test Expectation on Multiple-Choice Performance and Sujective Ratings. Teaching Psychology, 34(4), 219-225. doi: 10.1080/00986280701700094

Bentler, P. M. (1990). Comparative fix indexes in structural models. Psychological Bulletin, 107, 238-246. doi: 10.1037/0033-2909.107.2.238

Bentler, P. M. (1995). EQS Structural Equations Program Manual. Encino, CA: Multivariate Software, Inc. Bentler, P.M. (2006). EQS Structural Equations Program Manual. Encino, CA: Multivariate Software, Inc. BOE. RD 1791/2010, de 30 de diciembre, por el que se aprueba el Estatuto del Estudiante Universitario.

Browne, M. W., \& Cudeck, R. (1993). Alternative ways of assessing model fit. In K. A. Bollen \& J. S. Long (Eds.), Testing Structural Equation Models. 132-162. Beverly Hills, CA: Sage. doi: $10.1177 / 0049124192021002005$

Burnett, P. C., \& Dart, B. C. (1997). Conventional versus confirmatory factor analysis: Methods for validating the structure of existing scales. Journal of Research and Development in Education, 30, 126-132.

Doménech, F. (2011). Evaluar e investigar en la situación educativa universitaria. Un nuevo enfoque desde el Espacio Europeo de Educación Superior. Publicacions de la Universitat Jaume I, Universitas, 34.

Doménech, F. (2013). Un Modelo Instruccional para Guiar la Reflexión y la Investigación en el Aula: El Modelo de Calidad de Situación Educativa. Electronic Journal of Research in Educational Psychology, 11(1), 239-260

Doménech, F., Fortea, M.A., \& Benaloy, M. (2013). Cuestionario de evaluación inicial del Sistema de evaluación de la asignatura. En IV Jornada Nacional sobre Estudios Universitarios. El futuro de los 
títulos universitarios. Castellón: Servicio de Publicaciones de la Universitat Jaume I.

Entwistle, N. J. (1987). Understanding classroom learning. London: Hodder and Stoughton. (Traducción castellana: La comprensión del aprendizaje en el aula. Barcelona Paidós, MEC).

European Association for Quality Assurance in Higher Education (ENQA) (2005). Standards and guidelines for Quality Assurance in the European Higher Education Area. Informe traducido por la ANECA: “Criterios y Directrices para la Garantía de Calidad en el Espacio Europeo de Educación Superior”.

Hoe, S. L., (2008). Issues and procedures in adopting structural equation modeling thechnique. Journal of Applied Quantitative Methods, 3, 76-83.

Hoyle, R. H. (1995). The structural equation modeling approach: Basic concepts and fundamental issues. In R. H. Hoyle (Ed.), Structural equation modeling, concepts, issues, and applications (pp. 1-15). Thousand Oaks, CA: Sage.

López, V.M. (coord) (2009). Evaluación Formativa y Compartida en Educación Superior. Propuestas, técnicas, instrumentos y experiencias. Madrid: Narcea.

Maclellan, E. (2001). Assessment for learning: the differing perceptions of tutors and students. Assessment and Evaluation in Higher Education, 26(4). 307-318. Doi: 10.1080/02602930120063466

Marton, F. (1988). Describing and Improving Learning. In R. Schmeck (Ed.), Learning strategies and learning styles, 53-82. New York: Plenum Press. doi: 10.1007/978-1-4899-2118-5_3

Marton, F \& Saljo, R. (1976) On qualitative differences in learning: I-Outcome and process. British Journal of Educational Psychology, 46, 4-11. doi: 10.1111/j.2044-8279.1976.tb02980.x

Nunnaly, J. C., \& Berstein, I. H. (1994). Psychometric Theory, (3 ${ }^{\text {rd }}$ edition). McGraw-Hill, New York. doi: $10.1177 / 073428299901700307$

Padilla, M. T. \& Gil, J. (2008). La evaluación orientada al aprendizaje en la Educación Superior: condiciones y estrategias para su aplicación en la docencia universitaria. Revista Española de Pedagogía, 241, 467486.

SanMartí; N. (2007). Evaluar para aprender: Diez ideas clave. Barcelona. Graó.

Scouller, K. M. (1998). The influence of assessment method on students' learning approaches: Multiple choice question examination versus assignment essay study practices of Asian students. Higher Education, 40, 99-121. doi:10.1023/A:1003196224280

Snow, R., \& Swanson, J. (1992). Instructional Psychology. Annual Review of Psychology, 43, 583-626. doi:10.1146/annurev.ps.43.020192.003055

Struyven, K., Dochy, F., \& Janssens, S. (2005). Students' perceptions about evaluation and assessment in higher education: A review. Assessment and Evaluation in Higher Education, 30(4), 331-347. doi:10.1080/02602930500099102

Tian, X. (2007). Do assessment methods matter? A sensitivity test. Assessment \& Evaluation in Higher Education, 32(4), 387-401. doi:10.1080/02602930600898460

Trillo, F., \& Porto, M. (1999). La percepción de los estudiantes sobre su evaluación en la universidad. Innovación educativa, 9, 55-75

Zabalza, M. A. (2003). Competencias docentes del profesorado universitario. Madrid: Narcea. 
Anexo: Cuestionario

\section{Cuestionario de evaluación de la percepción inicial de los estudiantes universitarios sobre el sistema de evaluación de la asignatura" (CEPISEA).}

Cuestionario para el/la Estudiante

ASIGNATURA:

TITULACIÓN:

Curso:

Edad:

Hombre

Mujer $\square$
¿Qué se evalúa?: La información facilitada al estudiante sobre el sistema de evaluación y calificación de la asignatura.
¿Cuándo se evalúa?: Al inicio del curso
¿Para qué se evalúa?: Para detectar deficiencias y corregirlas
¿Quién evalúa?: El/la estudiante

Uno de los requisitos más importantes de la docencia universitaria conforme al Espacio Europeo de Educación Superior y del respeto a los derechos de los estudiantes es que la información inicial sobre la asignatura, que proporciona la universidad y su profesorado, sea clara, completa, comprensible y transparente. Para lograr una docencia de calidad y una fructífera relación de enseñanza/aprendizaje, es fundamental que el estudiante conozca y comprenda desde el inicio de curso que es lo que se espera de él para aprobar la asignatura o incluso obtener una buena calificación.

Por ello, se te pide de forma anónima, que una vez ya te han presentado el sistema de evaluación y calificación de la asignatura con sus criterios y condiciones correspondientes, y a la vista de la guía docente o programa de la asignatura, de las explicaciones facilitadas por el profesorado, de la información contenida en el aula virtual y cualquier otro recurso o material propio del curso, valores los siguientes aspectos de la evaluación.

\section{INSTRUCCIONES}

1. Procura no dejarte ningún ítem sin contestar.

2. No debes comentar tus respuestas con los compañeros.

3. Al finalizar el cuestionario se te pedirá que nos indiques, según tu opinión, cómo se podría mejorar el sistema de evaluación de la asignatura.

4. Sé sincero en tus contestaciones.

5. Si no entiendes algo o te surge alguna duda mientras estás cumplimentando el cuestionario, levanta el brazo para que te podamos ayudar.

6. Para puntuar cada una de las afirmaciones debes utilizar siempre el mismo sistema de respuesta:

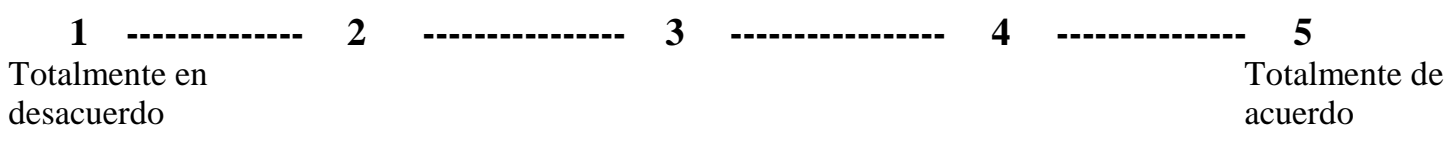




\begin{tabular}{|c|c|c|}
\hline \multicolumn{2}{|r|}{$\begin{array}{l}\text { El programa oficial o guía docente ECTS de la asignatura (publicado en el LLeu)* } \\
\text { proporciona suficiente información sobre... }\end{array}$} & $\begin{array}{l}\text { Evaluación } \\
\text { (de } 1 \text { a 5) }\end{array}$ \\
\hline 1 & La estrategia de evaluación y calificación & \\
\hline 2 & El tipo y volumen de prueba/s de examen. & \\
\hline 3 & $\begin{array}{l}\text { Las condiciones y requisitos que debe cumplir el estudiante para tener derecho a realizar } \\
\text { los exámenes y ser calificado. }\end{array}$ & \\
\hline 4 & $\begin{array}{l}\text { Los criterios y mínimos exigibles para aprobar las diferentes pruebas de examen y la } \\
\text { asignatura en global. }\end{array}$ & \\
\hline 5 & Los criterios que se aplicarán para calcular la calificación final de la asignatura. & \\
\hline 6 & $\begin{array}{l}\text { Los criterios de recuperación respecto a los exámenes y otras tareas evaluables } \\
\text { suspendidas. }\end{array}$ & \\
\hline $7 * *$ & $\begin{array}{l}\text { Las ausencias, enfermedades u otras circunstancias atenuantes de la evaluación de los } \\
\text { estudiantes. }\end{array}$ & \\
\hline 8 & Cómo será la evaluación y calificación en la segunda convocatoria del curso. & \\
\hline \multicolumn{2}{|r|}{$\begin{array}{l}\text { El profesor/a se ha esforzado por transmitir y explicar al estudiante el sistema de } \\
\text { evaluación de la asignatura..... }\end{array}$} & $\begin{array}{l}\text { Evaluación } \\
\text { (de } 1 \text { a } 5 \text { ) }\end{array}$ \\
\hline 9 & Facilitando la información fundamental desde principio de curso. & \\
\hline 10 & $\begin{array}{l}\text { Disponiéndola en un lugar público fácilmente accesible y localizable para el estudiante } \\
\text { (aula virtual, reprografía, etc.). }\end{array}$ & \\
\hline 11 & $\begin{array}{l}\text { Presentando en clase con suficiente detalle el sistema de evaluación y calificación al } \\
\text { estudiante. }\end{array}$ & \\
\hline 12 & $\begin{array}{l}\text { Resolviendo y aclarando satisfactoriamente las dudas del estudiante sobre el sistema de } \\
\text { evaluación y calificación. }\end{array}$ & \\
\hline 13 & $\begin{array}{l}\text { Justificando al estudiante por qué se usan determinados procesos evaluadores y no otros } \\
\text { diferentes. }\end{array}$ & \\
\hline 14 & $\begin{array}{l}\text { Utilizando tanto recursos presenciales (clase, tutoría, etc.), como telemáticos (e-mail, aula } \\
\text { virtual, Google apps, etc.). }\end{array}$ & \\
\hline 15 & $\begin{array}{l}\text { Mostrándose accesible para que los estudiantes le puedan consultar cualquier duda o } \\
\text { aclaración. }\end{array}$ & \\
\hline
\end{tabular}

Cómo se percibe al inicio del curso el sistema de evaluación. La evaluación que se propone para esta asignatura me parece...

(de 1 a 5$)$

16 Transparente y comprensible, es decir, se entiende con claridad como va a ser evaluado el estudiante.

17 Realista, pues, tal y como está planteada, parece coherente con el número de

17 horas/créditos de la asignatura, temario a impartir, tamaño de la clase, etc.

18 Razonable en cuanto al nivel de exigencia para superar la materia (es decir, ni demasiado fácil, ni demasiado difícil).

19 Motivante, ya que anima al estudiante a llevar la asignatura al día y a esforzarse.

20 Justa, pues es correcto el peso o porcentaje que se otorga a las diferentes pruebas (exámenes, trabajos, prácticas, etc.) para la obtención de la nota final.

21 Facilitadora del aprendizaje, en el sentido que va a ayudar al estudiante a organizar y dosificar su esfuerzo a lo largo del curso.

22 Fiable, es decir, permitirá valorar los aprendizajes del estudiante con acierto y precisión.

23 Válida, en el sentido de que es adecuada para evaluar los aprendizajes propios de la 23 materia.

24 Objetiva, ya que su concreción y operatividad permitirá reducir la subjetividad e interpretación personal del docente al valorar los aprendizajes alcanzados.

25 Personalizable, ya que permite que el estudiante elija entre diferentes tareas, pruebas y/o procedimientos de evaluación adaptadas a sus necesidades concretas.

* El Lleu es el lugar donde se publica la información oficial de los estudios oficiales de nuestra universidad.

** Basándonos en los resultados obtenidos, el ítem 7 ha sido eliminado. 